\title{
VISITA DOMICILIAR A UM PORTADOR DE TRANSTORNO DO ESPECTRO AUTISTA VIA INTEGRAÇÃO ENSINO-SERVIÇO-COMUNIDADE NA ATENÇÃO PRIMÁRIA A SAÚDE: ESTUDO DE CASO
}

\author{
HOME VISIT CARRIER OF THE AUTISTIC SPECTRUM DISORDES VIA \\ INTEGRATION TEACHING-SERVICE-COMMUNITY IN THE PRIMARY \\ ATTENTION TO HEALTH: CASE STUDY
}

Raí Novaes Nogueira, Aniele Pereira Lima, Kátia Novaes dos Santos, Vinicius Santiago Alves, Stela Almeida Aragão, Thainan Alves Silva, Zenilda Nogueira Sales

Universidade Estadual do Sudoeste da Bahia - UESB

\begin{abstract}
It is common for the primary care health professional to find, in different types of care, pathological conditions that involve a subjective dimension of mental health problems. In the field of attention to the child and adolescent population, neurodevelopmental disorders, among which, Autistic Spectrum Disorder (ASD) stands out among the most common conditions. In this study, an undergraduate experience of the medical course of the State University of Southwest of Bahia, in a situation of home visit and minor, is sought. TEA and major obesity carrier. It is hoped that this experience reported here will raise awareness about the difficulties faced by parents of ASD users, as well as difficulties in basic care to establish concrete interventions to the detriment of the parents' Mental health with Primary Care teams.
\end{abstract}

\begin{abstract}
Resumo
Comumente o profissional de saúde da assistência básica depara-se, em diversos tipos de atendimentos, com condições patológicas que envolvem uma dimensão subjetiva dos problemas da saúde mental. E no âmbito da atenção a população infanto-juvenil, os transtornos do neurodesenvolvimento, dentre os quais, o Transtorno do Espectro Autista (TEA), se destaca entre os quadros mais comuns. Neste estudo, procurou-se relatar a experiência de um graduando do curso de medicina da Universidade Estadual do Sudoeste da Bahia, numa situação de visita domiciliar a um menor com o TEA $e$ portador de obesidade importante. Espera-se que esta experiência aqui relatada, sensibilize $e$ difunda conhecimentos sobre as dificuldades enfrentadas por pais de indivíduos portadores de TEA, bem como, as dificuldades da atenção básica para se estabelecer intervenções concretas em detrimento, ainda, do parco apoio matricial de profissionais de saúde mental junto ás equipes da Atenção Básica.
\end{abstract}

Palavras chave: Transtornos do neurodesenvolvimento; Transtorno do espectro autista; Visita domiciliar; Obesidade
Key words: Neurodevelopmental Disorders; Autism spectrum disorder; Home visit; Obesity 


\section{Introdução}

Carentes de ações de cuidado, cerca de 31\% a $50 \%$ da população brasileira apresenta durante a vida pelo menos um episódio de algum transtorno mental, e $20 \%$ a $40 \%$ necessita, em decorrência desses transtornos, de algum tipo de ajuda profissional ${ }^{1}$. Dentre os transtornos mentais, considera-se que os transtornos iniciais do desenvolvimento do ser estão entre as enfermidades mais prevalentes ${ }^{1,2}$.

Segundo o DSM-V, 2014², o Transtorno do Espectro Autista (TEA) compreende um largo espectro de transtornos do neurodesenvolvimento que ocorre no período inicial da vida do ser humano. Caracteriza-se por déficits persistentes na comunicação social e na interação social e em habilidades para desenvolver, manter e compreender relacionamentos. Além dos déficits na comunicação social, o diagnóstico do TEA requer a presença de padrões restritos e repetitivos de comportamento, interesses e atividades, considerando que os sintomas mudam com o desenvolvimento ${ }^{2}$. De acordo com Gonzaléz $(2007)^{3}$, além das características mais marcantes percebidas no TEA, relacionadas principalmente ao falho desenvolvimento da linguagem e interação social, ainda há uma série de desordens gastrointestinais, com diminuída produção de enzimas digestivas, inflamação da parede intestinal, e a permeabilidade intestinal alterada $^{3}$. Segundo Abreu $(2011)^{4}$, crianças autistas possuem duas a três vezes mais chances de serem obesas do que a população em geral ${ }^{4}$. Aumento de peso foi associado a distúrbios do sono e uso de alimentos como recompensa. Houve também, uma tendência para associação da ingestão de risperidona e percentil de índice de massa corporal aumentada ${ }^{5,6,7}$. Todos esses fatores agravam os sintomas dos portadores do TEA.

A identificação de sinais iniciais do TEA ainda na fase inicial da infância possibilita a implementação imediata de ações importantes, uma vez que os resultados positivos em resposta a terapias são mais significativos quando instituídos precocemente ${ }^{8,9}$. Mediante a necessidade de um diagnóstico precoce, foi incluído na Atenção Básica e na Estratégia de Saúde da Família (ESF), pelo Ministério da Saúde, diretrizes gerais que remetem à dimensão subjetiva dos usuários e aos quadros mais graves de saúde mental, permitindo a identificação de problemas de saúde física e mental de crianças e adolescentes, enfatizando também o apoio ás famílias, a formação e treinamento das equipes da atenção básica para lidar com esta realidade e o apoio de profissionais de saúde mental junto a essas equipes ${ }^{9}$. Estas estratégias possibilitam maior acesso aos indivíduos portadores de transtornos mentais ao diagnóstico e assistência terapêutica precoce. Dentre as estratégias de aproximação, diálogo e vínculo, a visita domiciliar (VD) se insere como um instrumento de atenção à saúde, capaz de fortalecer e ampliar o olhar sobre as necessidades de saúde das pessoas, família e comunidade, possibilitando maior aproximação dos profissionais com os usuários e ampliando o reconhecimento do seu contexto de vida, condição básica para o cuidado integral $\left.\right|^{9,10,11,12}$

Contudo, cabe salientar que, nas ações dirigidas para pessoas com transtorno mental, muitos profissionais da assistência básica têm resistência e relata maior dificuldade em desenvolver ações de cuidado para essa população ${ }^{9,12}$. Isso sugere reflexões acerca da possível relação entre as ações em saúde mental, em detrimento da falta de conhecimento adequado para este cuidado, e a necessidade de se estabelecer uma relação de consciência e valorização sobre a importância da saúde mental no contexto da Atenção Básica e o melhor preparo acadêmico dos futuros profissionais no que tange ao tema em discussão. Neste contexto, a Faculdade de Medicina da Universidade Estadual do Sudoeste da Bahia, há uma década, vem implementando um currículo integrado e pautado em metodologias ativas de aprendizagem, pressupondo o processo de aprendizagem a partir da vivência da prática profissional. Esta proposta de currículo está pautada no conceito de integralidade do cuidado, sob a ótica das necessidades de saúde e de uma educação transformadora, crítica e reflexiva, estimulando a produção de conhecimento, promovendo a reflexão sobre a prática e, consequentemente, sua transformação.

Assim, propõe-se, neste relato, apresentar a experiência de um graduando em uma situação de visita domiciliar a um portador de TEA, realizada durante a atividade prática de disciplina de graduação.

\section{Metodologia}

A disciplina Prática de Integração Ensino/Serviço/Comunidade (PIESC), oferecida pelo curso de Graduação em Medicina da Universidade Estadual do Sudoeste da Bahia, requer como quesito para avaliação prática: a efetivação da aproximação dos graduandos com a comunidade a partir de visitas domiciliares a 
famílias que tenham um ou mais indivíduo(s) com agravo(s) a saúde física ou mental. O objetivo primordial da disciplina é aproximar o graduando em medicina da comunidade para que o mesmo possa fazer observações e posteriormente discutir as condições clínicas, socioeconômicas, culturais, ambientais e afetivas do indivíduo acometido no contexto familiar e intervir para promoção, prevenção e tratamento do agravo.

A partir da proposta da presente atividade prática, surgiu a ideia de descrever a experiência de um graduando, na companhia de um instrutor da PIESC e especialista em psiquiatra, em uma situação de visita a uma criança com TEA e sua família em domicílio. O motivo do primeiro encontro com a família foi através da sugestão de uma Agente Comunitária de Saúde (ACS), lotada em uma microárea do Programa de Saúde da Família (PSF) em uma zona periférica do município de Jequié/Bahia/Brasil. A atenção ocorreu devido a constante preocupação da genitora com o menor, expressa na solicitação de nossa visita, principalmente pela dificuldade em lidar com o quadro de ansiedade, agitação, insônia e compulsão alimentar, tendo a criança desenvolvido uma obesidade importante.

Uma semana após a sugestão pelo ACS, foi realizado o contato prévio através de uma primeira visita domiciliar do graduando e do instrutor do PIESC, para apresentação da proposta de atividade da disciplina. Trata-se de uma atividade prática da disciplina de graduação em medicina, tais como: visita domiciliar, exame clínico e comunicação terapêutica. A partir da concordância da genitora para a visita domiciliar, procedeu-se o agendamento da data e horário do encontro, bem como, do local, sendo acordado que seria em sua residência.

o local onde se dá o processo de comunicação é um fator importante e determinante de certos tipos de respostas ${ }^{13}$. Um ambiente conhecido e por vezes, de vivência, torna-se o melhor lugar em face da insegurança do desconhecido. Quando o paciente é visitado ou entrevistado num ambiente tranquilo, confortável e livre de pessoas interferindo, proporciona a sensação de se sentir mais à vontade para estabelecer uma situação comunicativa mais eficiente ${ }^{13,14}$.

Planejou-se a utilização de um questionário clínico, enfocando também as questões socioeconômicas e culturais, e informações para a genitora sobre o quadro de TEA de seu filho. A orientação se deu por um roteiro de avaliação neuropsiquiátrica embasado na literatura, tomando as precauções para que esse momento de interação não ficasse voltado somente à sequência do roteiro, mas que permitisse a comunicação livre e espontânea entre o entrevistador (graduando) e o entrevistado (genitora), para que essa comunicação pudesse ser efetiva.

Ao longo da trajetória acadêmica, o graduando/entrevistador realizou inúmeras visitas domiciliares, entretanto, cada visita possibilitou novas experimentações vivenciais, confirmando a cada novo encontro com uma família a importância singular da comunicação, e a necessidade de serem criadas estratégias para que a interação e a comunicação sejam produtivas para todos que participam dos diálogos. Stefanelli ${ }^{13}$ observa ainda que, através da comunicação, são compartilhadas informações de interesse comum, podendo a mesma ser verbal ou não verbal. Nesse processo, existe uma interação em contínuo movimento: cada momento de comunicação é único e não se repete $^{13}$. Para Paulo Freire $^{10}$, o diálogo é condição essencial para os sujeitos atingirem um estágio de consciência devotada à responsabilidade política e social ${ }^{10}$.

\section{Contextualizando a experiência}

$\mathrm{Na}$ visita domiciliar realizada à família, no dia 04 de Julho de 2016 ás 15 h, foi estabelecido o segundo contato e comunicação com um núcleo familiar composto por um casal e seus dois filhos. Pode-se verificar diversos aspectos da vida desta família. Dentre estes aspectos, o comportamento de D.M., um infante de 5 anos de idade, diagnosticado portador de Transtorno do Espectro Autista (TEA), um transtorno localizado no grupo dos transtornos do neurodesenvolvimento, comprometendo de certo modo a autonomia do mesmo, e tornandoo dependente dos cuidados de sua genitora e demais familiares. A visita traduziu-se, ao mesmo tempo, na coleta de dados e discussão de estratégias de intervenção no âmbito da assistência básica ao indivíduo com agravo a saúde física e mental e também oportunizou ao acadêmico e ao médico/instrutor, a possibilidade de contribuir com a família, oferecendo incialmente, informações sobre o TEA e orientações para a melhoria do cuidado. $\mathrm{Na}$ ocasião, o graduando e o instrutor foram recepcionados pela genitora de D.M. com muita cordialidade, tendo sido um momento singular de aprendizado. Num diálogo franco e amistoso, pudemos tomar conhecimento da condição de vida de modo geral dos seus filhos, em especial de D.M. e também pudemos contribuir com 
orientações de grande valia para a genitora, que demonstrou satisfação em nos receber.

A genitora iniciou o diálogo nos relatando que D.M. teve seu primeiro ano de vida com um desenvolvimento psicomotor e neurossensorial superior eficiente. $\mathrm{O}$ menor andou aos 11 meses e começou a balbuciar as primeiras palavras aos 1 ano e 2 meses. Até este período, parecia tudo ocorrer em normalidade. No entanto, por volta dos 1 ano e 5 meses, surgiram algumas alterações comportamentais como agitação excessiva, involução da fala e algum tempo depois, evoluindo com uma perda de controles esfincterianos, com evacuações sem indiciar sinais de desconforto durante o ato, o que seria uma característica de interação não-verbal ou mesmo verbal (choro) de uma criança na altura da idade apresentada, isto é, desenvolver mecanismos que informam ao seu cuidador para os devidos cuidados. Outros aspectos notados pela genitora, foi a pobreza de expressões gestuais e faciais, movimentos estereotipados e sem objetivo, sendo algumas vezes rítmicos e repetitivos, dificultando a interação social e com o mundo em volta, ainda que, para os mais próximos, houvesse sinais de afeto. Todos esses sinais levaram a família a suspeitar que alguma desordem estava ocorrendo com o filho D.M.

Mediante os sinais observados, a genitora procurou o serviço clínico inicialmente no posto de saúde da família da localidade, que referenciou o menor ao Centro de Atenção Psicossocial (CAPS) da cidade, e através de um serviço especializado de psicologia e psiquiatria, foi recomendado que D.M. fosse consultado por um neuropediatra, que avaliou o caso e deu o diagnóstico de TEA. Segundo a genitora, foi difícil compreender e aceitar o diagnóstico e a certeza da condição do filho, pois havia um temor e uma auto concepção de que a patologia enquadraria seu filho como um "louco" e com tão pouca idade. Após muitos diálogos com psicólogos do CAPS, agentes sociais e mesmo da Associação de Pais e Amigos de Excepcionais (APAE), a genitora tomou conhecimento de que o quadro do seu filho se tratava de um transtorno do desenvolvimento, que se inicia precocemente e evolui cronicamente. $E$ que ao longo deste processo evolutivo, outras potencialidades poderiam se desenvolver, bem como as possibilidades de melhora clínica e funcional ao longo do tempo.

O diagnóstico de TEA permanece essencialmente clínico e é feito a partir de observações da criança e entrevistas com pais e/ou cuidadores ${ }^{1,2,8,9,12,15}$. A identificação em casos de sinais iniciais de problemas de desenvolvimento que podem estar futuramente associados com características e histórico clínico sugestivo de TEA, prestigia intervenções extremamente importantes, uma vez que os resultados favoráveis em resposta a terapias são tão mais significativos quanto mais precocemente instituídos $2,8,9,12$.

Dando seguimento ao relato, na ocasião do diagnóstico, D.M. estava com dois anos e quatro meses. Como conduta terapêutica, prescrita pelo médico neuropediatra, risperidona ${ }^{\circledR}$ de $1 \mathrm{mg}$ e clonazepam (Rivotril ${ }^{\circledR}$ ) Gotas $1 \mathrm{mg}$ (equivalente a 10 gotas), os quais proporcionaram uma melhora do quadro de impulsividade e da instabilidade psicomotora e afetiva. No entanto, após três meses em uso do esquema terapêutico abordado, a genitora notou que D.M. mantinha irritabilidade e insônia diariamente. Em nova consulta, após alguns meses, foi necessário substituir o fármaco Rivotril ${ }^{\circledR}$ pelo cloridrato de levomepromazina (Neozine ${ }^{\circledR}$ ) Gotas 4\%, 20mg (20 gotas) pela manhã e 40mg (40 gotas) á noite, mantendo uso da risperidona, com modificação da posologia para $2 \mathrm{mg} /$ dia em duas tomadas em horários regulares. Atualmente, o menor tem adormecido por períodos mais longos á noite, pelo efeito sedativo, além da ação neuroléptica do Neozine ${ }^{\circledR}$. No entanto, devido aos efeitos colaterais da risperidona, a criança vem apresentando compulsão alimentar, significativo ganho de peso e obesidade. Relata ainda que, D.M. já realizou diversas sessões de fonoaudiologia, porém, sem apresentar sinais de reabilitação da linguagem.

$\mathrm{Na}$ atualidade, ainda não há cura para os principais sintomas do autismo. No entanto, vários grupos de fármacos, incluindo neurolépticos típicos e atípicos, foram usados para tratar problemas comportamentais associados, como agressão, comportamentos auto-prejudiciais e mesmo o quadro de agitação ${ }^{16}$. Os antipsicóticos demonstram um benefício no tratamento de condutas agressivas ou autolesivas de pessoas com TEA, quando há baixa resposta ou não adesão às intervenções não medicamentosas, muitas vezes devido à própria gravidade do comportamento ${ }^{16,17}$.

Outros aspectos foram discutidos ao longo da visita a família. O primeiro ponto se refere ao suporte que D.M. recebe no seu tratamento. A genitora nos informou que o posto de saúde da localidade não oferece nenhum suporte aos cuidados de sua criança especial, sendo que o único contato apenas para a vacinação. Quanto aos serviços prestados pelo CAPS, refere que a criança estava matriculada e recebendo o serviço da instituição, no entanto, a genitora nos relatou 
que, devido a procura muito grande das pessoas por este serviço, existe uma falta de suporte que acredita ser necessário para o cuidado adequado e aprendizado de seu filho, tornando a atenção um recurso humano não tão presente. Portanto, não tem levado mais o D.M. ao CAPS.

Ao mesmo tempo, além do cuidado à saúde no âmbito da atenção especializada, que objetivam responder às especificidades clínicas de suma importância, é importante também ressaltar que os serviços de saúde devem funcionar em rede, estando preparados para acolher e responder às necessidades gerais de saúde da pessoa com TEA, o que inclui o acompanhamento básico e especializado, tanto da equipe de habilitação/reabilitação, quanto da equipe multidisciplinar ${ }^{9,12}$. É também de fundamental importância que a pessoa com TEA receba, no decorrer da vida, ações e programas no âmbito da proteção social, da educação, do lazer, da cultura, dentre outras ações com a finalidade do cuidado integral e da promoção de uma melhor qualidade de vida ${ }^{9,12,18,19}$.

Conforme a genitora, toda a família se organiza para dá todo o suporte necessário para D.M. O mesmo recebe benefício de prestação continuada (LOAS), sendo utilizado este recurso para custear com os cuidados necessários como manutenção de despesas com consultas médicas periódicas, exames complementares, medicações, fonoaudiólogos, alimentação, dentre outros recursos. Ao genitor, cumpri a função mantenedora do ponto de vista financeiro, porém, sempre está presente e contribuindo na medida do possível na atenção aos filhos. A filha de 10 anos contribui na dinâmica do cuidado do irmão mais em muitas situações cobra atenção dos pais, e notadamente da genitora.

A genitora relatou que sua filha tem contribuído bastante nos cuidados com o irmão, porém, desabafou sobre sua preocupação em não estar provendo os devidos cuidados para com a mesma, uma vez que sua atenção se desvia para o ato de cuidar do filho com TEA. No diálogo, alertamos para a genitora que a garota logo precisará de mais atenção, que entrará em fase de adolescência, viverá a maturação sexual, e as primeiras experiências de um novo ciclo biológico, social, e que todos esses aspectos estarão intrinsecamente relacionados com a formação psicológica da filha. Necessitará de orientações quanto a fase da menarca, pubarca, telarca, etc. Todos, eventos marcantes na vida de uma mulher, onde será imprescindível orientações, diálogos, atenção e proximidade.

Para a genitora, cumpri a ela o papel de maior responsabilidade com D.M. e quase todas as suas tarefas diárias são direcionadas ao mesmo. Nos relatou que não tem disponibilidade de tempo para um trabalho ou cumprir qualquer compromisso com carga horária regular e continuada. Também informou que já teve vínculos de trabalho, sendo que no último, teve que solicitar desvinculo devido a demanda do cuidado do filho.

Os cuidados diários concedidos à pessoa com TEA exige da família longos e permanentes períodos de dedicação, promovendo, em muitos casos, a restrição do tempo para outras atividades de trabalho, lazer e até de descuido com a saúde dos demais membros da família, o que tem se relacionado aos níveis elevados de tensão física e psicológica, e na qualidade de vida das mães ${ }^{9,12,18,19}$. Isto significa que é imprescindível para a família se desenvolver, mesmo na presença de um evento estressor como no caso dos problemas de saúde e/ou de desenvolvimento dos filhos, também de espaços de escuta, de proventos e de uma rede de apoio e sobretudo, na qualidade das relações entre os membros da família ${ }^{6,9,12,14,18,19}$.

Seguindo o instrumento para realização da visita domiciliar, foi realizado também o exame clínico de D.M. com os seguintes achados: Ectoscopia: Em bom estado geral, pouco colaborativo, o que impediu a realização de determinadas etapas do exame. Desorientado no tempo e espaço e halopsiquicamente. Antropometria: Peso: $40 \mathrm{~kg}$; altura: $1.13 \mathrm{~cm}$. Segundo a Cartilha de Acompanhamento do Crescimento e Desenvolvimento (Programa CD), preconizada para avaliação do crescimento e desenvolvimento da criança e os valores antropométricos colhidos no exame de D.M., pudemos determinar que o menor se encontra com altura dentro dos parâmetros de normalidade, entretanto com peso significativamente acima dos parâmetros esperados para a idade preconizados pela OMS (Organização Mundial de Saúde) e MS (Ministério da Saúde). D.M. se enquadra na curva de peso $x$ idade com peso notadamente acima do peso máximo esperado para sua idade ${ }^{9,12}$. Avaliação cardiorrespiratória sem anormalidades. Abdômen indolor a palpação. A genitora aproveitou a ocasião para informar que seu filho tem feito muita ingesta de carboidratos e rejeita as frutas, suco e verduras.

Crianças autistas têm um padrão alimentar e um estilo de vida diferente das crianças não autistas, comprometendo seu crescimento corporal, bem como seu estado nutricional ${ }^{5}$. 0 comportamento repetitivo e interesse restrito 
podem ter papel importante na seletividade dietética ${ }^{6}$. Segundo Abreu (2011) ${ }^{4}$, as crianças autistas possuem duas ou três vezes mais chances de serem obesas do que a população em geral. No TEA, mudanças de peso foram associadas a distúrbios do sono e uso de alimentos como recompensa ${ }^{4}$.

$\mathrm{Na}$ sucinta avaliação neuropsiquiátrica, detectou-se humor não polarizado, psicomotricidade aumentada e agitação; atenção espontânea reduzida e atenção voluntária e afetividade preservada. Não apresentava alteração de sensopercepção; linguagem não desenvolvida, emite alguns sons e gritos. Funções executivas superiores como juízo de realidade alterados. Perda da consciência de controle das funções fisiológicas como a micção (D.M. se urinou durante o período da visita); não emitia sinais de desconforto ou alerta ao cuidador.

Mediante os relatos expostos, a história clínica de D.M. e o exame clínico realizado no mesmo, e considerando os aspectos patológicos que vem sendo assistido com as devidas condutas terapêuticas expostas no decorrer do presente relato de experiência, acentuamos a discussão no entorno da investigação do quadro de obesidade que a criança vem apresentando. Sendo para tal, uma das possíveis explicações o uso do fármaco risperidona e seus efeitos colaterais. Estudos evidenciaram uma provável associação da ingesta de risperidona e percentil de IMC mais alto em pacientes com TEA. O aumento do apetite e as alterações estão entre os efeitos mais comuns do uso da risperidona ${ }^{6,7}$.

A genitora foi orientada pelo graduando quanto à importância da inclusão da atividade física diária (caminhada) para D.M., da manutenção regular do sono do mesmo sempre que for possível (dormir um número de horas suficiente para o seu padrão de sono) e da adoção de alimentação equilibrada, condutas necessárias para ajudar a reverter o quadro de obesidade de D.M., e na próxima consulta agendada para o psiquiatra, relatar o ganho ponderal de D.M. para o profissional e buscar junto ao mesmo, alternativas fármacoterapêuticas à risperidona, se for possível.

\section{Conclusão}

A Visita Domiciliar (VD), ao longo de sua história, vem sendo praticada por profissionais médicos, enfermeiros, e por pessoas leigas treinadas para esta finalidade. $O$ sentido de tais visitas sempre esteve mais voltado para o controle de doenças e minimizar o sofrimento do que propriamente em promover a saúde, considerando o contexto social e a qualidade de vida. $\mathrm{Na}$ atualidade, a VD se constitui em um caminho para formar os profissionais de saúde com a nova visão do processo saúde/doença, além da revelação das possíveis necessidades de ajuste.

Esta experiência de visita domiciliar reafirmou que os profissionais da saúde, ainda nos dias de hoje, necessitam voltar a sua atenção ao ser humano, compreendendo suas características físicas e mentais e seu contexto social, econômico, cultural, as relações familiares e comunitárias, dentre outros aspectos.

O médico e demais profissionais de saúde, ainda carecem em nosso tempo de avanço das ciências, de um olhar mais holístico e humanizado. Neste contexto, se faz necessário a adoção de uma prática mais eficaz das ações promotoras da saúde mental. A Atenção aos casos psiquiátricos precisa estar fundamentada na formação clínica básica do futuro profissional médico, o que remete à necessidade de coerência entre o ensino e sua efetivação na prática.

A Atenção Básica deve estar preparada para atender e desenvolver ações essenciais com um portador de transtorno mental, seja ele transtornos do neurodesenvolvimento ou de outra natureza psiquiátrica. É indispensável também, o apoio aos familiares. Ofertar aos pais e cuidadores, espaços de escuta, acolhimento e de orientações, visando o empoderamento das famílias para a superação dos estigmas, para a busca de ferramentas que lhes auxiliem no cuidado àquele indivíduo portador da enfermidade mental.

Em nossa experiência, infelizmente, tivemos um exemplo de uma ação deficitária das instituições que prestam assistência básica ao indivíduo portador de um agravo à saúde mental, e que, embora a assistência especializada se faça presente institucionalmente, não produz uma Atenção com a qualidade e eficiência necessária.

Espera-se que este estudo sensibilize e promova a disseminação de conhecimentos sobre o TEA a partir do reconhecimento dos sinais clínicos do mesmo, o empoderamento das famílias, contribuindo para a superação do estigma no entorno desta doença, da ainda premente falta de informação e da gestão ineficaz aos cuidados e Atenção a um indivíduo portador do autismo. 


\section{Referências}

1. Dalgalarrondo P. Psicologia e semiologia dos transtornos mentais. 1. ed. Porto Alegre-RS: Artmed, 2000.

2. Diagnostic and Statistical Manual of Mental disorders - DSM-5. 5th.ed. Washington: American Psychiatric Association, 2013.

3. Gonzaléz E. e colaboradores.
específicas/intervenção psicoeducacional. Porto Alegre-RS: Artmed, 2007.

4. Abreu LC. Condições relacionadas a obesidade secundária na interface do crescimento e desenvolvimento. Rev. Brasileira de crescimento e desenvolvimento humano. São Paulo-SP, 2011

5. Zuchetto AT, Miranda TB. Estado nutricional de crianças e adolescentes, EFDeportes.com, Revista digital, Ano 16, n.156, Buenos Aires, May, 2011.

6. Silva $\mathrm{N}$ I. Relações entre hábito alimentar e síndrome do espectro autista. São Paulo: USP, 2011. Tese [Dissertação de Mestrado em Ciência e Tecnologia de Alimentos] Programa de Pós-Graduação em Ciência e Tecnologia de Alimentos, Escola Superior de Agricultura Luis de Queiroz, Universidade de São Paulo, São Paulo, 2011.

7. Kummer A, Barbosa IG, Rodrigues DH, Rocha NP, Rafael MDS, Pfeilsticker $L$, et al. Frequency of overweight and obesity in children and adolescents with autism and attention deficit/hyperactivity disorder. São Paulo: Revista Paulista de Pediatria: Orgão Oficial da Sociedade de Pediatria de Sao Paulo. 2016;34:71-7.

8. BARBARO J.; RIDGWAY, R. N.; DISSANAYAKE, C. Developmental surveillance of infants and toddlers by maternal and child nurses in na Australian community - based setting: promoting the early identification of autism spectrum disorders. J. Pediatr. Rio J, 2011.

9. Brasil. Ministério da Saúde. Diretrizes de Atenção à Reabilitação da Pessoa com Transtorno do Espectro Autista (TEA). Brasília: MS. 2014.

10. Mandú ENT, Gaiva MAM, Silva MA, Silva AMN. Visita domiciliária sob o olhar do usuário do programa de saúde da família. Texto e Contexto Enferm, 2008; 17(1):131-140.

11. Marin MJS, Gomes R, Junior ACS, Nunes CRR, Cardoso CP, Otani MP, Moravcik MY. O sentido da visita domiciliária realizada por estudantes de medicina e enfermagem: um estudo qualitativo com usuários de unidades de saúde da família. Ciência e Saúde, 2011; 16(11): 4357-65.

12. Brasil. Ministério da Saúde. Caderneta da Saúde da Criança: Menino. Brasília, DF, 2013.

13. Stefanelli MC. Comunicação com paciente: Teoria e ensaio. São Paulo: Robe Editorial. 1993.

14. Silva MJP. da. Comunicação tem remédio: A comunicação nas relações interpessoais em saúde. São Paulo: Robe Editorial. 1996.

15. Bair $\mathrm{G}$ et al. Prevalence of disorders of the autism spectrum in a population cohort of children in South Thames: the special needs and autism project (SNAP). The Lancet, [S.I.], v. 368, p. 210-215, 2006.

16. McCracken JT, McGough J, Shah B, Cronin P, Hong D, Aman MG, et al. Risperidone in children with autism and serious behavioral problems. N Engl J Med. 2002;347:314-21.

17. Weissman L., Bridgemohan C. Autism spectrum disorder in children and adolescents: Behavioral and educational interventions. In: Augustyn M, Patterson MC, Torchia MM, editors. UpToDate. Version 23. Waltham: Wolters Kluwer Health; 2015.

18. Semensato M, Schmidt C., Bosa C. Grupo de familiares de pessoas com autismo: relatos de experiências parentais. Aletheia, n.32, p.183-194, 2010.

19. Marques MH, Dixe MAR. Crianças e jovens autistas: impacto na dinâmica familiar e pessoal de seus pais. Rev. psiquiatr. clín. [online]. 2011. Disponível em: (Acessado em 11/Set/2015).

\section{Endereço para Correspondência}

Universidade Estadual do Sudoeste da Bahia UESB

Rua José Moreira Sobrinho - Jequiezinho, Jequié BA

CEP:. 45206-190

e-mail: rainovaes@hotmail.com

Recebido em 16/02/2018

Aprovado em 02/05/2018

Publicado em 30/06/2018 